\title{
EXIT Chart Analysis for Turbo LDS-OFDM Receivers
}

\author{
Razieh Razavi, Muhammad Ali Imran and Rahim Tafazolli \\ Centre for Communication Systems Research \\ University of Surrey \\ Guildford GU2 7XH, Surrey, U.K. \\ Email:\{R.Razavi, M.Imran, R.Tafazolli\}@surrey.ac.uk
}

\begin{abstract}
In this paper, the mutual information transfer characteristics of turbo Multiuser Detector (MUD) for a novel air interface scheme, called Low Density Signature Orthogonal Frequency Division Multiplexing (LDS-OFDM) are investigated using Extrinsic Information Transfer (EXIT) charts. LDSOFDM uses Low Density Signature structure for spreading the data symbols in frequency domain. This technique benefits from frequency diversity besides its ability of supporting parallel data streams more than the number of subcarriers (overloaded condition). The turbo MUD couples the data symbols' detector of LDS scheme with users' FEC (Forward Error Correction) decoders through the message passing principle. The effect of overloading on LDS scheme's performance is evaluated using EXIT chart. The results show that at $E_{b} / N_{0}$ as low as 0.3, LDS-OFDM can support loads up to $300 \%$.
\end{abstract}

Index Terms-Low density signature, Multiuser detection, Iterative decoding.

\section{INTRODUCTION}

In recent years, there has been considerable interest on improving the efficiency of modulation and coding techniques to be used for broadband wireless services. Future wireless communication systems are expected to provide a range of high speed services with different Quality of Service (QoS) requirements. In this regard Orthogonal Frequency Division Multiplexing (OFDM) as a multi-carrier system is deemed to be a useful approach to cope with wideband service demands due to its capability of exploiting both time and frequency resources as well as interference shaping property [1]. By dividing a wideband fading channel into flat narrow band channels, OFDM allows performing a high data rate transmission while avoiding inter-symbol interference due to channel's frequency selectivity [2].

OFDMA (Orthogonal Frequency Division Multiple Access) is an efficient extension of OFDM transmission to a multiuser communication scenario. In OFDMA systems, the set of subcarriers is divided into several mutually exclusive subsets that are assigned to different users for simultaneous transmission [3]. As in OFDMA, user-data symbols are assigned directly to sub-channels, the frequency domain diversity will not be achievable at modulation symbol level. Thus this will be crucial to incorporate properly designed error correction coding and interleaving schemes to obtain this diversity at a later stage [4].
LDS-OFDM approach combines benefits of OFDM based multi-carrier transmission with a recent idea on Low Density Signature (LDS) based spreading proposed for CDMA (Code Division Multiple Access) systems in [5]. In LDS-OFDM, due to low density signature structure, every data symbol will only be spread over a small subset of subcarriers (effective processing gain) and also every subcarrier will only be used by a small subset of data symbols that could belong to different users. The LDS structure can be captured by a low density graph, thus, similar to the application of LDS for CDMA system, the detection of LDS-OFDM could be based on message passing algorithm (MPA) presented in [5] for LDS-CDMA systems. This new technique can be viewed as a system which applies LDS as multiple access technique and OFDM for multi-carrier modulation. In other words, LDSOFDM is a special case of MC-CDMA (Multi Carrier-CDMA) which its signature is spars matrix. In [6], it was shown that LDS-OFDM could improve the performance if compared to OFDMA but with the cost of increased complexity. It is noticeable that the complexity of LDS-OFDM is higher than the conventional OFDMA but it is still affordable. In [7] sets of spreading sequences that are specifically designed to suit a belief-propagation multiuser detection structure were presented. It is shown that the performance improvements can be achieved using this structured approach if compared to the LDS with random signatures.

Inspired by the message passing analysis presented in [8] [9], this paper evaluates the extrinsic information transfer characteristics to describe the flow of information through the soft-input soft-output (SISO) components of the turbo MUD for the LDS-OFDM scheme. The Extrinsic Information Transfer chart (EXIT chart) is used for finding the effect of loading on convergence of turbo MUD which give us insights about the maximum load that the LDS structure can tolerate.

The LDS-OFDM's turbo receiver is made of two basic components: LDS multiuser symbol detector and a bank of users' SISO FEC decoders. The extrinsic information is iteratively exchanged between the two components towards a solution with less number of bit errors. Noticeable performance improvement is observed compared to the conventional receiver that does not benefit from this iterative exchange of information. EXIT chart analysis is employed to evaluate 
the convergence of the considered turbo MUD [10]. For both of its components, a detection/decoding trajectory is derived to visualize the evolution of extrinsic information in their detection/decoding process. Simulation results suggest that the derived EXIT charts are able to accurately predict the convergence behavior of the turbo MUD used for LDS-OFDM.

This paper is organized as follows: Section II presents the LDS-OFDM system architecture. Section III depicts the EXIT chart analysis of the turbo MUD of LDS-OFDM. Numerical results and analysis are provided in Section IV. Conclusions are drawn in section $\mathrm{V}$.

\section{LDS-OFDM SYSTEM MODEL}

In the LDS-OFDM technique, the original data streams are first multiplied with their low density spreading sequences and then modulated on different sub-carriers. The conceptual block diagram of an uplink LDS-OFDM system is shown in Fig. 1. We consider LDS-OFDM system with $K$ users and user indices $k=1, \ldots, K$.

Without loss of generality all users are assumed to take their symbols from the same binary constellation alphabet $\mathcal{X} \in\{ \pm 1\}^{1}$. Also users are assumed to have the same number of data symbols $M$ so the spreading signature for user $k$ will be $\mathbf{S}_{k}=\left(\mathbf{s}_{k, 1}, \ldots, \mathbf{s}_{k, M}\right) \in \mathbb{C}^{N_{c} \times M}$ that has only $d_{v}$ nonzero components on each column. We define $d_{v}$ as the effective spreading factor. Let also define $\mathbf{S}=\left(\mathbf{S}_{1}, \ldots, \mathbf{S}_{K}\right) \in \mathbb{C}^{N_{c} \times M K}$ as the overall low density signature matrix. Also $d_{c}$ is defined as the number of symbols that are allowed to interfere to each other at each chip. Let $\mathbf{A}=\operatorname{diag}\left(A_{1}, \ldots, A_{K}\right)$ and $\mathbf{G}_{k}=\operatorname{diag}\left(g_{k, 1}, \ldots, g_{k, N_{c}}\right)$ represent the users' transmit gain and the corresponding channel gain for user $k$ respectively. Each user's generated chip will be transmitted over a subcarrier of OFDM system. Then the received spreading signature for data symbol $m$ of user $k$ will be $\mathbf{h}_{k, m}=A_{k} \mathbf{G}_{K} \mathbf{s}_{k, m}$. In particular, the received signature at chip $n$ of data symbol $m$ of user $k$ is $h_{k, m}^{n}=A_{k} g_{k, n} s_{k, m}^{n}$.

To further explain and clarify, in this system, each chip represents a subcarrier of OFDM modulation and the data symbols using the same subcarrier will interfere with each other. The amount of interference will depend on the allocated power of data symbols on each subcarrier and user's corresponding channel gain.

Let $\mathcal{J}_{n}=\left\{(k, m): s_{k, m}^{n} \neq 0\right\}$ be the set of different users' data symbols that share the same chip $n$ or in other words be the set of nonzero positions in the $n^{\text {th }}$ row of the signature matrix S. So the received signal at $n^{\text {th }}$ chip (subcarrier) can be written as:

$$
y_{n}=\sum_{(k, m) \in \mathcal{J}_{n}} h_{k, m}^{n} x_{k, m}+v_{n},
$$

where $v_{n}$ is the additive white Gaussian noise of subcarrier $n$ and $x_{k, m}$ is the $m^{t h}$ data symbol of user $k$. At the receiver

\footnotetext{
${ }^{1}$ It can be extended to any modulation order but here for the convenience of presentation we assume BPSK modulation.
}

side, after performing OFDM demodulation operations the signal is passed to a near-optimum MUD based on Message Passing Algorithm (MPA) [5]. An LDS system with $K$ users and $N$ chips can be shown using factor graph $\mathcal{G}(\mathcal{U}, \mathcal{C})$ where users' symbols are represented by variable nodes $u \in \mathcal{U}$ and chips are represented by function nodes $c \in \mathcal{C}$. The connections between the received chip and its related users are represented by edges. The basic form of chip level iterated MUD can be explained as follows. Using MPA, messages containing the reliability values of adjacent variable node are exchanged between the function nodes and the variable nodes. The messages of the $j^{\text {th }}$ iteration sent by variable nodes are updated using the following rule:

$$
L_{l, \text { out }}^{j}=\sum_{m \neq l} L_{m, \text { in }}^{j-1},
$$

where $L_{m, i n}$ is the $m^{\text {th }}$ a priori L-value (Log Likelihood Ratio) going into variable node, $L_{l, \text { out }}$ is the $l^{\text {th }}$ extrinsic L-value coming out of the variable node. For $n^{\text {th }}$ function node the message of $j^{\text {th }}$ iteration is calculated as follow:

$$
L_{l, \text { out }}^{j}=f\left(x_{l} \mid y_{n}, L_{m, \text { in }}^{j}, \forall m \in \mathcal{J}_{n} \backslash\{l\}\right) .
$$

To approximate the optimum MAP detector, the function $f(\cdot)$ in (3) represents marginalization function. This function calculates extrinsic values for all the constituents bits involved in (1), based on (1) and observed chip $y_{n}$ and a priori input information. Having a small number of interferers in each subcarrier allows applying Maximum A Posteriori based Chip-Level iterated (MAP-CLi) multiuser detection [5]. After appropriate number of iterations the soft output which is the calculated log likelihood ratio at each variable node will be sent to the channel decoders. More details regarding the LDS MUD can be found in [5].

The turbo receiver of LDS-OFDM is based on iterative detection/decoding between LDS data symbol detector and users' FEC decoders. This is realized by iterative exchange of extrinsic information between detection and FEC decoding stages. The block diagram of LDS-OFDM's turbo MUD is illustrated in Fig. 1. In the turbo MUD, there exist two iterative processes: inner and outer iterative processing for MUD and the turbo-style processing, respectively. Considering now that two iterative processes are involved, the message update algorithm should be addressed accordingly. Let $t$ be the turbo iteration index, for inner iteration the message updates in (2) and (3) can be modified as:

$$
\begin{gathered}
L_{l, \text { out }}^{j, t}=\sum_{m \neq l} L_{m, \text { in }}^{j-1, t}+L_{d, \text { out }}^{t-1}, \\
L_{l, \text { out }}^{j}=f\left(x_{l} \mid y_{n}, L_{m, \text { in }}^{j, t}, \forall m \in \mathcal{J}_{n} \backslash\{l\}\right),
\end{gathered}
$$

where $L_{d, \text { out }}^{t-1}$ is the extrinsic information coming from an FEC decoder to its corresponding variable node in the previous 


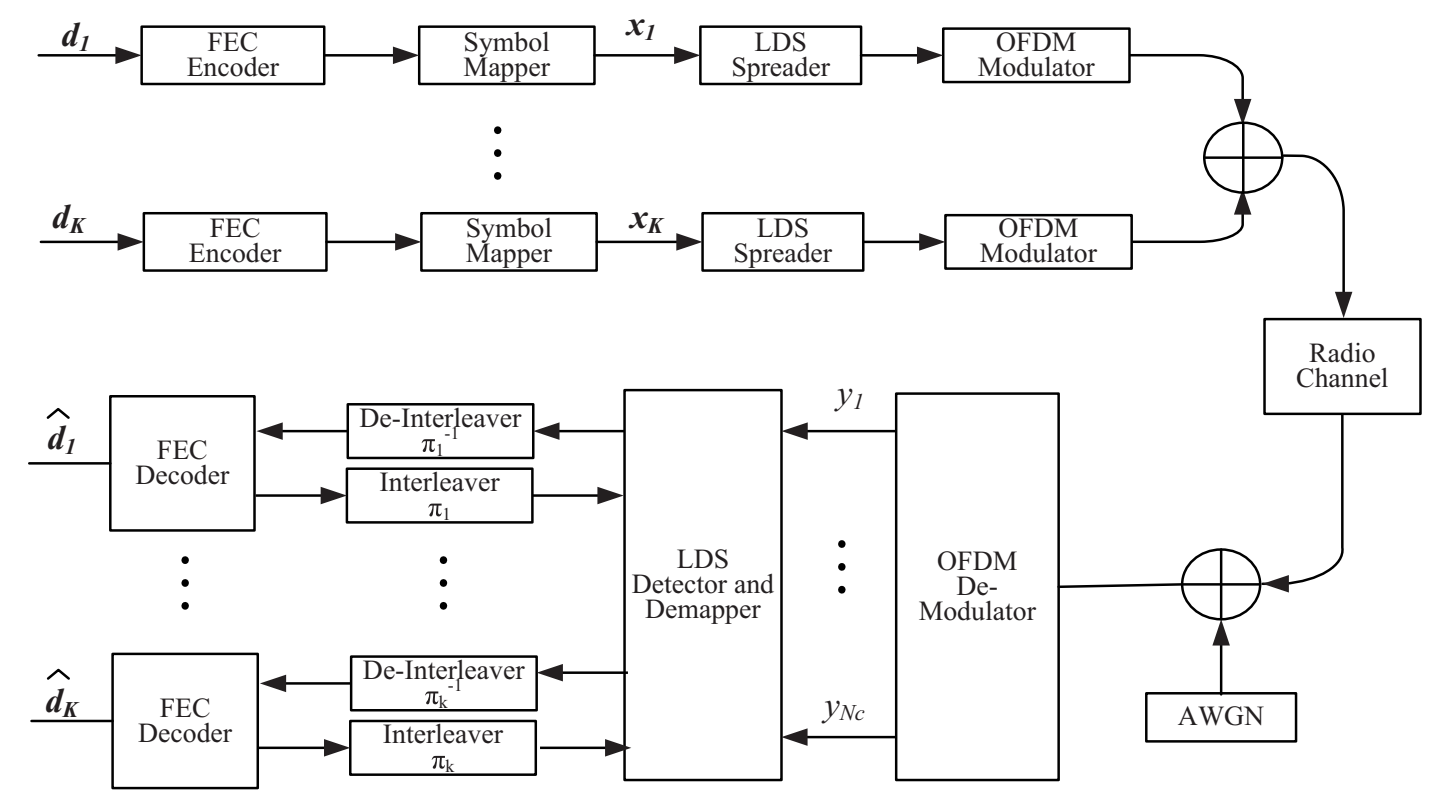

Fig. 1. LDS-OFDM block diagram.

turbo iteration. So considering message passing algorithm [11] the messages that variable nodes send to decoder are as follows:

$$
L_{v, \text { out }}^{j, t}=\sum_{m \neq l} L_{m, i n}^{j-1, t}
$$

As mentioned earlier $m$ represents the index of all the function nodes connected to the variable node. In other words the variable node with degree $d_{v}$ has $d_{v}+1$ incoming messages; $d_{v}$ from function nodes and 1 from decoder which gives a priori $\mathrm{L}$-value about variable node. Following message passing rule for the new graph, the variable node must consider the messages it receives from all the connected function nodes when calculating its message to be sent out to its corresponding FEC decoder. On the other hand when sending message to a function node it must consider all the messages it has received from the rest of the connected function nodes plus the message that has come from the corresponding FEC decoder. The message update for function nodes remain the same as for conventional LDS-OFDM MUD.

\section{EXIT Chart AnAlysis of Turbo Multiuser DETECTOR}

Extrinsic Information Transfer (EXIT) chart is a useful tool to analyse the information transfer between the two components of a decoder with iterations. Inspired by the message passing analysis, in this section the extrinsic information transfer characteristics are evaluated to describe the flow of information through the Soft-Input Soft-Output (SISO) components of the turbo multiuser detection algorithm for the turbo receiver of LDS-OFDM scheme. So the extrinsic L-values are passed on and interpreted as a priori information by the other detector or decoder. We use the notation of [8] and write $I_{A}$ for the average mutual information between the bits sent to the detector/decoder (which are the bits about which extrinsic L-values are exchanged) and the $a$ priori L-values. Similarly, $I_{E}$ refers to the average mutual information between the bits sent to the detector/decoder and the extrinsic L-values.

In order to compute an EXIT function, $L_{m, i n}^{j-1}$ in (6) is modelled as the output L-value of an AWGN channel whose input is the $m^{t h}$ interleaver bit transmitted using BPSK. So referring to [8], we can write a priori L-value by applying an independent Gaussian random variable $n_{A}$ with variance $\sigma_{A}^{2}$ and mean zero in conjunction with known bits on the detector graph edges $x \in \pm 1$ as follows:

$$
A=\mu_{A} x+n_{A}
$$

where

$$
\mu_{A}=\frac{\sigma_{A}^{2}}{2}
$$

So the mutual information $I_{A}=I(X ; A)$ is calculated as follows [8]:

$$
\begin{aligned}
& I_{A}=\frac{1}{2} \sum_{x=-1,1} \int_{-\infty}^{+\infty} p_{A}(\xi \mid X=x) \\
& \quad \log _{2} \frac{2 p_{A}(\xi \mid X=x)}{p_{A}(\xi \mid X=-1)+p_{A}(\xi \mid X=1)} d \xi .
\end{aligned}
$$

Considering that the conditional probability density function $p_{A}(\xi \mid X=x)$ is related to L-value $A$, with Gaussian distribution and with properties mentioned in (6) we will have: 
TABLE I

$$
\begin{aligned}
& I_{A}\left(\sigma_{A}\right)=1- \\
& \quad \int_{-\infty}^{+\infty} \frac{e^{-\left(\left(\xi-\sigma_{A}^{2} / 2\right)^{2} / 2 \sigma_{A}^{2}\right)}}{\sqrt{2 \pi} \sigma_{A}} \log _{2}\left[1+e^{-\xi}\right] d \xi .
\end{aligned}
$$

For abbreviation we define:

$$
J(\sigma):=I_{A}\left(\sigma_{A}=\sigma\right),
$$

with

$$
\lim _{\sigma \rightarrow 0} J(\sigma)=0, \quad \lim _{\sigma \rightarrow \infty} J(\sigma)=1, \quad \sigma \geq 0 .
$$

Therefor, to create the EXIT chart, a constituent decoder (either MUD or FEC decoder) is modelled as a device, mapping a sequence of observations and the input a priori information to a new sequence of extrinsic information. So the mutual information for both MUD and decoder can be calculated by, firstly, estimating the PDF from the histogram of the output L-values of a constituent decoder and then using (9) we can calculate the mutual information numerically. In line with [10], the EXIT chart can also be used to predict the BER performance considering that the soft output of the coded bits can be estimated by summing up the a priori information and the extrinsic information, which is denoted as:

$$
\begin{gathered}
\Lambda=L_{M U D}+L_{D E C}, \\
\sigma_{\Lambda}^{2}=\sigma_{L_{M U D}}^{2}+\sigma_{L_{D E C}}^{2},
\end{gathered}
$$

where (13) assumes independence between a priori information and the extrinsic information. Both variances are obtained from their corresponding mutual information using (11). The BER then can be calculated as follow:

$$
p_{b} \approx \frac{1}{2} \operatorname{erfc}\left(\sqrt{\frac{\mu_{\Lambda}^{2}}{\sigma_{\Lambda}^{2}}}\right)=\frac{1}{2} \operatorname{erfc}\left(\frac{\sigma_{\Lambda}}{2}\right) .
$$

Hence, using EXIT chart analysis we will be able to find the maximum load that the LDS-OFDM system can handle for a target BER.

\section{Simulation Results}

In this section, we present the simulation results for LDS-OFDM systems over different loading conditions. LDSOFDM's signatures are generated randomly. The simulation parameters are listed in Table I. Since the individual users' signals interfere with each other and influence each other's $a$ priori mutual information $I_{A}$ and extrinsic mutual informa-

\begin{tabular}{|c|c|c|}
\hline \multicolumn{2}{|c|}{ Number of Users } & 10 \\
\hline \multicolumn{2}{|c|}{ Number of data sub-channels } & 60 \\
\hline \multicolumn{2}{|c|}{ FFT size } & 64 \\
\hline \multicolumn{2}{|c|}{ Multipath channel model } & ITU Pedestrian Channel B \\
\hline \multicolumn{2}{|c|}{ Channel coding } & Half-rate convolutional code \\
\hline \multicolumn{2}{|c|}{ Modulation } & BPSK \\
\hline \multirow{4}{*}{ Data streams per user } & 200\% Loading & 12 \\
\hline & $250 \%$ Loading & 15 \\
\hline & $300 \%$ Loading & 18 \\
\hline & $350 \%$ Loading & 21 \\
\hline \multicolumn{2}{|c|}{ Effective spreading factor (LDS) } & $d_{v}=3$ \\
\hline
\end{tabular}
tion contributions $I_{E}$, an $K$-dimensional EXIT chart would be required. In order to circumvent this problem and hence allow us to plot the EXIT curves for a multi-user communication system in a two-dimensional, rather than $K$-dimensional plane, the average of all the user mutual information is calculated.
Simulation PARAMETERS

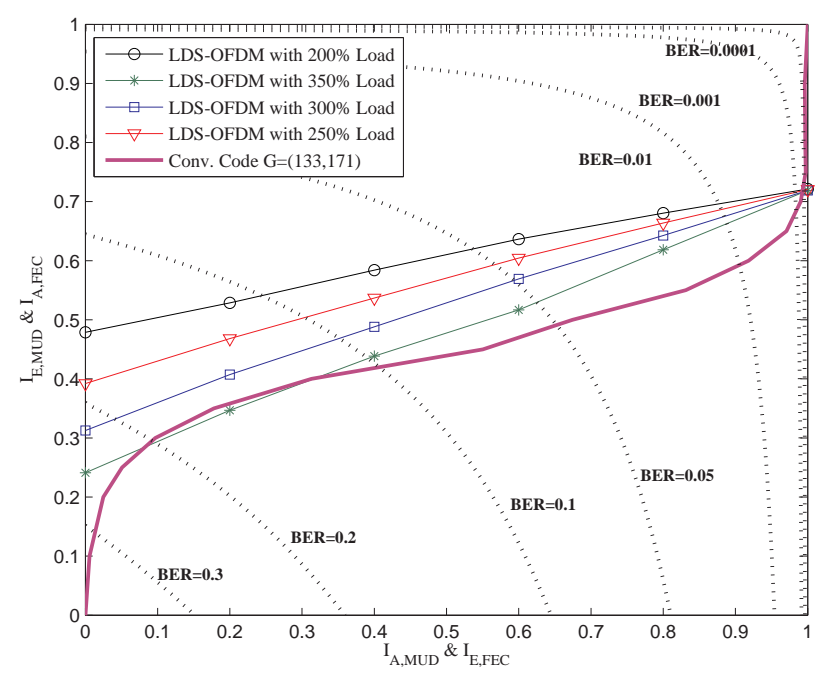

Fig. 2. EXIT chart for turbo MUD at $E_{b} / N_{0}=0.3 \mathrm{~dB}$ for AWGN channel.

We denote the a priori information and extrinsic information of the MUD by $I_{A, M U D}$ and $I_{E, M U D}$, respectively, while the corresponding quantities of the channel decoder by $I_{A, F E C}$ and $I_{E, F E C}$, respectively. Fig. 2 illustrates the EXIT chart for the turbo MUD with different loaded condition over AWGN channel. The iterative process starts with $I_{A, M U D}=0$ which means no prior information for the MUD. Next, the output L-values are fed into decoder while their output L-values are then fed back to MUD and so forth. Considering the mentioned results we can say that LDS-OFDM is able to tolerate up to $300 \%$ load at $E_{b} / N_{0}$ as low as $0.3 \mathrm{~dB}$. For the $350 \%$ loaded case, the two curves intersect at low mutual information level which result in high BER. Fig. 2 also shows that the curves related to MUD for different overloaded conditions meet at $I_{A}=1$, which is because for perfect a priori information, the MUD is able to remove the multiple access interference (MAI) completely and overloading effect will be cancelled.

Fig. 3 illustrates the EXIT chart for the turbo MUD with different loaded condition over a typical multipath fading 


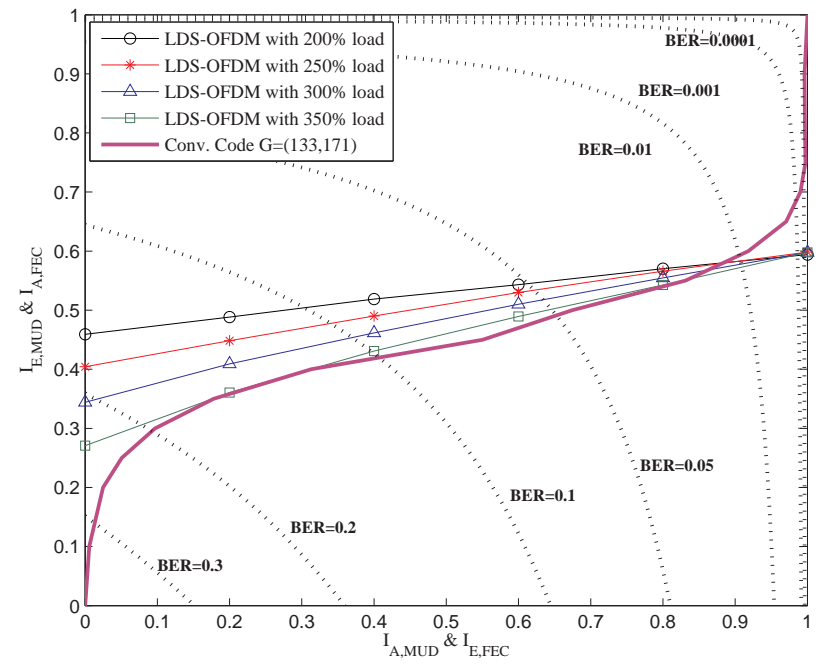

Fig. 3. EXIT chart for turbo MUD at $E_{b} / N_{0}=0.3 \mathrm{~dB}$ for multipath fading channel.

channel. It shows at $E_{b} / N_{0}=0.3 \mathrm{~dB}$ the maximum load the LDS-OFDM system can handle is $300 \%$ as the curves intersect at early staged for load $350 \%$, so the receiver will not converge at appropriate level of mutual information. Finally Fig. 4 reveals the effect of SNR on the convergence behavior of LDS-OFDM. It is noticeable that an increase in the SNR merely related to a vertical shift of MUD curve towards higher extrinsic output. It is also shown that the simulated decoding trajectory evolves within the open detection tunnel between the EXIT curves of the MUD and the channel decoder, until it reaches the intersection of the curves. Since the simulated detection trajectories closely follow the EXIT curves of the receiver components, the validity of EXIT chart analysis can be verified. The analysis can be extended to higher order modulations. So a better spectral efficiency can be achieved by finding the trade-off on tuning modulation level and loading factor.

\section{CONCLUSIONS}

Increasing interest in high data rate services demands high spectral efficiency. In this regard LDS-OFDM has recently been introduced as an efficient multiple access technique. In order to increase the spectral efficiency of LDS-OFDM, the loading or modulation order must be increased while keeping the performance near single user bound. However, this requires efficient MUD receiver to decode overloaded parallel data streams. To keep the complexity low these receivers usually operate on iterative decoding principle and their convergence analysis becomes important. In this paper we provide the framework and tools to perform this analysis.

By analysing the convergence behavior of the turbo MUD, we were able to show how loading affects the performance as the curves intersect at points where mutual information is lower for higher loading values. The results shows that at

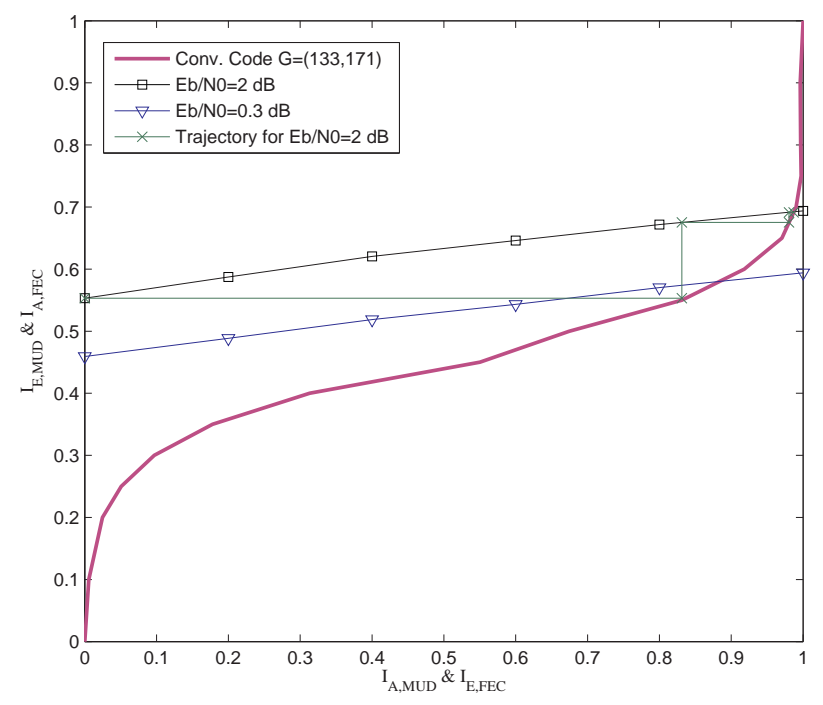

Fig. 4. EXIT chart for turbo MUD for $200 \%$ load under multipath fading channel.

$E_{b} / N_{0}$ as low as $0.3 \mathrm{~dB}$, the receiver of LDS-OFDM is able to detect parallel data streams up to $300 \%$ more than the number of subcarriers.

\section{ACKNOWLEDGMENT}

This work was supported by Huawei Technologies Co., Ltd, China.

\section{REFERENCES}

[1] A. Pandharipande and S. Dasgupta, "Optimum multiuser OFDM systems with unequal subchannel assignment", in Proc. IEEE Int. Conf. Communications ICC '03, 2003, vol. 5, pp. 3423-3427.

[2] R. V. Nee and R. Prasad, OFDM for wireless multimedia communications, Artech House, 2000.

[3] L. Hongxiang and L. Hui, "An analysis of uplink OFDMA optimality", IEEE Transactions on Wireless Communications, vol. 6, no. 8, pp. 29722983, 2007.

[4] D. Tse and P. Viswanath, Fundamentals of Wireless Communication, Cambridge, 2005.

[5] R. Hoshyar, F. P. Wathan, and R. Tafazolli, "Novel low-density signature for synchronous CDMA systems over AWGN channel", IEEE Transactions on Signal Processing, vol. 56, no. 4, pp. 1616-1626, 2008.

[6] R. Hoshyar, R. Razavi, and M. Al-Imari, "LDS-OFDM an efficient multiple access technique", in Proc. IEEE 71st Vehicular Technology Conf. (VTC 2010-Spring), 2010, pp. 1-5.

[7] J. van de Beek and B. M. Popovic, "Multiple access with lowdensity signatures", in Proc. IEEE Global Telecommunications Conf. GLOBECOM 2009, 2009, pp. 1-6.

[8] S. Ten Brink, "Convergence behavior of iteratively decoded parallel concatenated codes", IEEE Transactions on Communications, vol. 49, no. 10, pp. 1727-1737, 2001.

[9] S. Ten Brink, G. Kramer, and A. Ashikhmin, "Design of low-density parity-check codes for modulation and detection", IEEE Transactions on Communications, vol. 52, no. 4, pp. 670-678, 2004.

[10] L. Kai and W. Xiaodong, "EXIT chart analysis of turbo multiuser detection", IEEE Transactions on Wireless Communications, vol. 4, no. 1, pp. 300-311, 2005.

[11] F. R. Kschischang and B. J. Frey, "Iterative decoding of compound codes by probability propagation in graphical models", IEEE Journal on Selected Areas in Communications, vol. 16, no. 2, pp. 219-230, 1998. 